\title{
Search for Muon-to-Electron Conversion at J-PARC: COMET Experiment
}

\author{
Hajime Nishiguchi* \\ High Energy Accelerator Research Organization (KEK) \\ E-mail: hajime.nishiquchidkek.ip
}

The COMET Experiment at J-PARC aims to search for the lepton flavour violating (LFV) process of muon-to-electron conversion in a muonic atom, $\mu^{-} N \rightarrow \mathrm{e}^{-} N$, with a branching-ratio sensitivity of $6 \times 10^{-17}$ which is 4 orders of magnitude better than the present upper limit.

Complemental searches for two kinds of muon LFV decay modes, $\mu^{+} \rightarrow \mathrm{e}^{+} \gamma$ and $\mu^{-} N \rightarrow \mathrm{e}^{-} N$, are quite important in order to explore the parameter region predicted by most well-motivated theoretical models beyond the Standard Model such as SUSY-GUT, seesaw, little-Higgs, extradimension, etc. MEG collaboration reported the latest result on $\mu^{+} \rightarrow \mathrm{e}^{+} \gamma$ search at PSI in Switzerland, and made a strong limit on new physics models, i.e. filling in the missing peace of muon LFV, $\mu^{-} N \rightarrow \mathrm{e}^{-} N$, will certainly play a very important role. The need for such an unprecedented sensitivity places several stringent requirements on both the muon beam and the detector system.

In order to realise the experiment effectively and efficiently, a staging approach to deployment is endorsed by the J-PARC Program Advisory Committee and KEK, and the COMET Phase-I experiment will commence its engineering runs in 2019. In Phase-I, a search for $\mu^{-} N \rightarrow \mathrm{e}^{-} N$ will be performed with an intermediate sensitivity which is 100 times better than the present upper limit.

In this contribution, prospects for COMET Phase-I and Phase-II both are presented in addition to the current status of construction of facility and detectors.

The European Physical Society Conference on High Energy Physics

5-12 July, 2017

Venice

\footnotetext{
*Speaker.

${ }^{\dagger}$ On behalf of the COMET Collaboration
} 


\section{Muon-to-Electron Conversion}

\subsection{Physics Motivation}

A Lepton Flavour Violation (LFV) among charged leptons, eg. $\mu^{-} N \rightarrow \mathrm{e}^{-} N$ process etc., which has never been observed while the quark mixing and the neutrino oscillations have been experimentally confirmed, is attracting a great deal of attention, since its observation is highly expected by most of well-motivated theories beyond the Standard Mode[W]. It is predicted that $\mu^{-} N \rightarrow \mathrm{e}^{-} N$ is naturally causable with a branching ratio just below the current experimental upper bound, $10^{-13} \sim 10^{-16}$, by leading theories for physics beyond the standard model, e.g. Supersymmetric theories of Grand Unification or Supersymmetric Standard Model with seesaw mechanism (eg. see Ref.[[]] for a review). The ambitious goal of the COMET experiment (COherent Muon to Electron Transition) [B] is searching for a $\mu^{-} N \rightarrow \mathrm{e}^{-} N$ process with an improved sensitivity by at least four orders of magnitude over the last best upper limit on a $\mu^{-} N \rightarrow \mathrm{e}^{-} N$ branching ratio reported by the SINDRUM-II collaboration, $7 \times 10^{-13}$ [四].

\subsection{Signature and Background}

The event signature of coherent $\mu^{-} N \rightarrow \mathrm{e}^{-} N$ conversion in a muonic atom is a monochromatic single electron emitted from the conversion with an energy of $E_{\mu \mathrm{e}}=m_{\mu}-B_{\mu}-E_{\text {recoil }}$, where $m_{\mu}$ is the muon mass, and $B_{\mu}$ is the binding energy of the $1 s$ muonic atom. $E_{\text {recoil }}$ is the nuclear recoil energy which is extremely small and negligible. Since $B_{\mu}$ depends on nuclei $E_{\mu \mathrm{e}}$ varies, $e g$. 104.3 MeV for Ti and 105.1 MeV for Al.

There are several potential sources of electron background events in the energy region around $100 \mathrm{MeV}$, which can be grouped into three categories as follows. The first group is intrinsic physics backgrounds which come from muons stopped in the muon-stopping target. The second is beamrelated backgrounds which are caused by beam particles of muons and other contaminated particles in a muon beam. The third is other backgrounds which are, for instance, cosmic-ray backgrounds, and fake tracking events, and so on.

In order to suppress backgrounds the following key elements have been proposed as the next generation of $\mu^{-} N \rightarrow \mathrm{e}^{-} N$ conversion search experiments.

(A) Beam Pulsing: Since muonic atoms have lifetimes of the order of $1 \mu \mathrm{sec}$, a pulsed beam with its width that is enough short compared with these lifetimes would allow one to remove beam-related backgrounds by performing measurements in a delayed DAQ window. To eliminate prompt beam-related backgrounds, excellent extinction of proton beam is required during the DAQ interval.

(B) Pion Capture System with a High Solenoid Field: Superconducting solenoidal magnets of a high magnetic field surrounding a proton target to capture pions in a large solid angle, called pion capture system, leads to a dramatic increase of muon yields by several orders of magnitude.

(C) Curved Solenoids for Muon Transport: The curved solenoids are adopted to select charges and momenta of muons as well as removing neutral particles in a beam. In a curved solenoidal magnetic field, a centre of the helical trajectory of a charged particle is shifted perpendicular to the curved plane. The shift, whose amount is given as a function of momentum and its charge, makes a dispersive beam. 
The COMET experiment[[3] at the Japan Proton Accelerator Research Complex (J-PARC) was proposed in order to realize above very innovative ideas.

\section{COMET Experiment}

\subsection{Experiment Overview}

The COMET uses a proton beam of $56 \mathrm{~kW}$ which will be available at J-PARC main ring (MR) accelerator. The aimed sensitivity at COMET is better than $10^{-16}$ which would explore most of the region predicted by leading theories for physics beyond the standard model. A schematic layout of the COMET experiment is presented in Figure $\mathrm{I}$. The COMET plans to use an $8 \mathrm{GeV}, 7 \mu \mathrm{A}$,

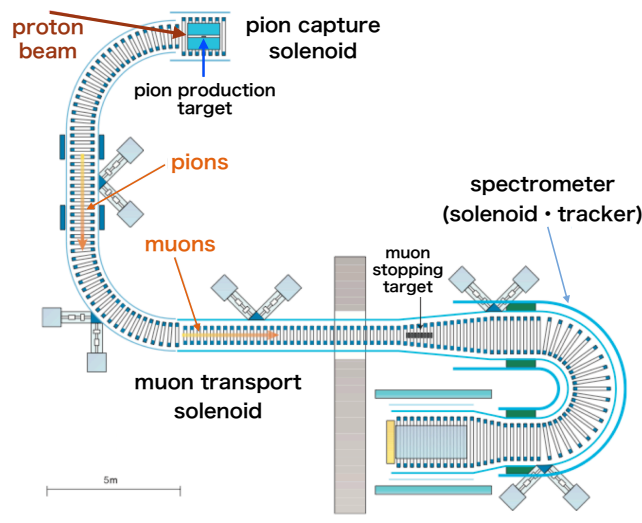

Figure 1: A schematic layout of the COMET experiment

slow extracted proton beam from the J-PARC MR. This equates to a beam power of $56 \mathrm{~kW}$ which is much less than the design power of $750 \mathrm{~kW}$. A proton beam is used to produce pions, which are then captured and transported by a series of superconducting solenoids. The pions decay into muons as they travel along the muon transport channel. The toroidal field of the muon transport channel selects muons with negative charge and momentum less than $75 \mathrm{MeV} / c$. The muon stopping target slows the muons down until they are captured in nuclear orbit by a nucleus in the target. A muon could then convert into an electron, with a momentum of around $105 \mathrm{MeV} / c$, or it could decay normally producing an electron with lower momentum. The electron spectrometer selects electrons of interest, collimating off electrons not of interest at the exit of the spectrometer. The momentum and energy of the electron is then measured by the tracker and calorimeter, respectively. The tracking detector is located in a uniform, $1 \mathrm{~T}$, magnetic field within the vacuum of the detector solenoid. It is designed to measure with good efficiency, the parameters of the helical trajectory of electrons that are emitted from the muon stopping target. The detector consists of a set of 5 straw tracker layers, $48 \mathrm{~cm}$ apart, and placed so that the axial direction of the straws is transverse to the axis of the solenoid. Each of the five tracker layers consists of four planes, two to measure the $x$ coordinate and two to measure the $y$ coordinate. Each pair of planes is staggered by half a straw diameter in order to solve left-right ambiguities. A calorimeter is positioned behind the tracker, and consists of $\mathrm{LYSO}\left(\mathrm{Lu}_{1.8} \mathrm{Y}_{0.2} \mathrm{SiO}_{5}(\mathrm{Ce})\right)$ crystal viewed by APD (avalanche photo diodes). Electrons 
which pass through the tracker are absorbed in the calorimeter and if the deposited energy lies within a window about $105 \mathrm{MeV}$, a trigger signal is generated.

\subsection{Staging Approach}

The COMET experiment is planned to be carried out separately by two stages, called "PhaseI" and "Phase-II". The COMET Phase-I will construct the pion-capture system and the curved solenoid up to the first $90^{\circ}$ bending and will place the detector at the exit of this first bending ahead of the "full COMET", called phase-II. There are two main purposes to perform COMET Phase-I prior to the Phase-II.

- Measure the beam at the $1 \mathrm{st} 90^{\circ}$ bending to understand the Background

- Perform the $\mu$ - e search at the 1 st $90^{\circ}$ bending with an intermediate sensitivity

Concerning the first purpose, so far the experimental optimization, not only for beam-line design but also for detector design, is completely based on the simulation. However, the hadron production code, eg. MARS[[] etc., needs to be verified with real data for such an extremely high dose environment. Phase-I background measurement will provide the significant input for such simulation framework, and give a further optimization for experiment which will guarantee the successful conclusion of experiment. Concerning the second purpose, even at the first $90^{\circ}$ bending one can perform the search experiment for $\mu^{-} N \rightarrow \mathrm{e}^{-} N$ conversion. Of course, this measurement cannot achieve the unprecedented sensitivity of final COMET experiment. However, one can achieve much better sensitivity than the current experimental upper bound, at least two orders of magnitude better than the current bound, thanks to the good pulsing and high intensity muon beam which is realized by J-PARC accelerator. For the $\mu^{-} N \rightarrow \mathrm{e}^{-} N$ search at Phase-I, Cylindrical Drift Chamber (CDC) system is used for the electron tracker in order to maximise the detector acceptance ${ }^{1}$.

\section{Current Status}

In the J-PARC hadron experimental facility, new proton beam line which is dedicated for the COMET experiment is under construction. The experimental hall for COMET experiment has been already completed and the cryostat system is under construction in the hall to maintain all the superconducting magnet system for COMET secondary beam line. Concerning the detector development, the CDC detector was completed for Phase-I and the commissioning run is ongoing using the cosmic ray. $\mathrm{R} \& \mathrm{D}$ for the straw tracker and the calorimeter for the beam measurement of Phase-I has been also recently completed, and the final assembly for them are just starting which will be completed in 2018. In addition to these detector systems, the diamond-based proton-beam monitor which is highly tolerant to a high power proton beam is under developed [可].

\footnotetext{
${ }^{1}$ Only $90^{\circ}$ bending and short transport line is not long enough to suppress severe backgrounds in the transported beam particles so that the centre part of the transported beam cannot be used for $\mu^{-} N \rightarrow \mathrm{e}^{-} N$ search experiment. In order to avoid a reduction of acceptance due to this issue, muon stopping target would be placed at the centre of tracking volume. This is the reason why Phase-I needs the cylindrical drift chamber.
} 


\section{Conclusion}

The COMET Experiment is searching for Muon-to-Electron Conversion which violates the lepton-flavour conservation. So far charged lepton-flavour violation (LFV) has never been observed while quark mixing and neutrino oscillations were experimentally confirmed. In order to explore the new physics beyond the Standard Model, searching for charged LFV is greatly attracted.

For this experiment, very high power and purely pulsed proton beam is necessary, and then this experiment was proposed to J-PARC (JAPAN Proton Accelerator Research Complex). The collaboration employed staging approach to realise the experiment quickly and effectively. In the first phase, called $\triangle$ COMET Phase-I" , beam measurement and $\mu$-e conversion search at an intermediate sensitivity will be conducted, and in Phase-II, $\mu$-e conversion search will be finally performed by the full sensitivity. Currently, detectors, facility, and beam line constructions are ongoing at a fever pitch. In early-2019, all the components will be ready to start its engineering run followed by the physics measurement.

\section{Acknowledgements}

This research is supported by the grant-in-aids, JSPS KAKENHI 16H02192 and 16H00876 (Japan).

\section{References}

[1] R. Barbieri and L. J. Hall, Phys. Lett. B338 (1994) pp.212-218

[2] T. Mori and W. Ootani, Prog. in Particle Nuclear Phys. 79 (2014) pp.57-94

[3] COMET Collaboration, Conceptual Design Report for Experimental Search for Lepton Flavor Violating $\mu^{-} N \rightarrow \mathrm{e}^{-} N$ Conversion at Sensitivity of $10^{16}$ with a Slow-Extracted Bunched Proton Beam (COMET) : J-PARC P21 (KEK Report 2009-10), 2009

[4] W. H. Bertl et.al.[SINDRUM-II Collaboration], Eur. Phys. J. C47 (2006) pp.337-338

[5] N.V.Mokhov, The MARS Code System User's Guide, Fermilab-FN-628 (1995)

[6] Y.Fujii, "Development of the Diamond based Proton Beam Monitor for COMET Experiment", (Presented in this conference) 